\title{
Introducing a new Educational Series: a step forward in Clinical and Translational Oncology dissemination
}

\author{
The Editorial Board
}

$\mathrm{O}^{\prime}$ ne of the most important tools to improve our chances of defeating cancer is to have better knowledge of how to deal with the disease. Clinical oncology has made tremendous progress in recent decades, continuously providing new and more successful ways to treat cancer patients. However, the amount of information generated is so overwhelming that it is almost impossible to follow individually, which makes it essential to devise mechanisms for proper and professional dissemination of the most significant and relevant improvements. Clinical and Translational Oncology is committed to provide all cancer researchers and health care professionals with the most upto-date information on patient management. In an effort to fulfil this commitment, in the present issue we are launching a new Educational Series to be called Yellow Series: Advances in clinical management and therapy of cancer. It is with pleasure that we introduce this new educational series, to be published every other month, in response to a spontaneous demand from many of our readers who have been asking for an educational section with a special focus on the clinical aspects of oncology practice. Currently, the three ongoing educational series in the journal, designated as Blue, Green and Red Series, are mainly oriented towards the molecular biology and basic research of cancer, with special focus on Translational Oncology.

This new series will complement those existing excellent series, which are highly appreciated by our readers and have been profusely requested to the authors, a good indication of their success. The yellow series will cover mainly aspects related to the clinical management of patients and practical therapy of cancer. The objective of the new series is to make room for as many different aspects of clinical management and multiple therapies of cancer as possible. So, we introduce this series with the intention of covering a wide range of issues, from very specific aspects of any kind of therapy or clinical management, to more general manuscripts like the one published in this issue of the journal.

This first manuscript is a guideline for the management of non-small-cell lung cancer (NSCLC), which was conceived and written by a panel of experts from the Spanish Society of Medical Oncology (SEOM). This will be the first of a series of guidelines that will be published periodically in this section of the journal. The objective of these guidelines is to provide an up-to-date and evidence-based recommendation for the clinical management of several cancers, in a user-friendly format. Obviously, it is not intended to perform a huge review or an extensive multidisciplinary statement, which is far from the objective of these clinical guidelines.

We hope that the new Yellow Series will be an ideal place for reviews dealing with specific aspects of new radiation therapies or surgery techniques, controversies about the management of different clinical stages of cancer, new procedures of diagnosis or advances in the medical treatment of different cancer subtypes.

We are convinced that this new series will be well received by our readers, will contribute to the growth of our journal and will be considered as a step forward in the dissemination of Clinical and Translational Oncology worldwide. It is written as part of our commitment to foster knowledge in a professional format to all those interested in cancer research and treatment. 